\title{
DE GRANT MAL AMALADIS \\ E LA PASTORELLA NASCOSTA \\ IN AUCASSIN ET NICOLETTE: UNA PROPOSTA DI DATAZIONE DELLA CHANTEFABLE*
}

\author{
Lucilla SPETIA \\ lucillaspetia@yahoo.it \\ Università dell'Aquila
}

\section{STATUS QUAESTIONIS: LA CONTROVERSA DATAZIONE DI AUCASSIN ET NICOLETTE}

L'anonima chantefable Aucassin et Nicolette 1 dalla particolare struttura fatta di lasse di eptasillabi assonanzati e prosa, costituisce senza dubbio uno dei testi più affascinanti ed enigmatici al tempo stesso della letteratura francese medievale.

Il suo fascino discende dall'atmosfera idilliaca dell'amore contrastato tra due giovani sul modello di Floire et Blanchefleur e di Pyramus et Thisbé, sovente invocati - con ogni probabilità, a ragione - come modelli di riferimento della chantefable, sia pure con modifiche anche sostanziali rispetto ad essi ${ }^{2}$. L'enigma - secondo una felice definizio-

\footnotetext{
${ }^{*}$ L'articolo è una riscrittura ampliata e ulteriormente sviluppata dell'intervento tenuto al XXVIII Congresso Internazionale della Società di Linguistica e Filologia Romanza svoltosi a Roma presso 1'Università della Sapienza dal 18 al 23 luglio 2016.

${ }^{1}$ Per il testo della chantefable si rinvia a Maria Antonia Liborio (ed.), Aucassin et Nicolette, Milano-Trento, Luni, 2001, che a p. 24 informa di essersi basata sull'edizione di Aucassin et Nicolette. Chantefable du XIII ${ }^{e}$ siècle, Mario Roques (ed.), Paris, Champion, 1963, apportando lievi modifiche.

${ }^{2}$ Per primo fu Edmond Faral, Recherches sur les sources latines des contes et romans courtois du Moyen Âge, Paris, Champion, 1913, pp. 26-33, ad accostare la chantefable a Piramus et Thisbé, nonostante le importanti differenze formali, perché mentre nel secondo testo domina il verso (couplets d'octosyllabes e diverse combinazioni metriche di carattere lirico), in Aucassin et Nicolette si osserva alternanza di versi e prosa, per cui cfr. infra; e quindi a sostenere che l'autore della chantefable avesse conosciuto Piramus et Thisbé. Invece Kaspar Rogger, «Étude descriptive de la chantefable Aucassin et Nicolette», Zeitschrift für romanische Philologie, 67 (1951), pp. 409-457, spec. pp. 435-436; e 70 (1954), pp. 1-58, spec. pp. 14 e 42-48, pur rilevando suggestioni narrative comuni tra i tre testi, non concorda con Faral poiché diversa è la lunghezza di Piramus et Thisbé e di Aucassin et Nicolette, e d'altra parte nella chantefable non si riscontra l'approfondimento psicologico proprio a Floire et Blanchefleur. Dal canto suo Giovanna Angeli, «Enfants, frères, amants: les ambiguités de l'idylle de Piramus et Thisbé à Aucassin et Nicolette», in Jean-Jacques Vincensini e Claudio Galderisi (dir.), Le Récit idyllique. Aux sources du roman moderne, Paris, Garnier, 2009, pp. 45-58, sostiene che i tre testi, insieme al lai Deus Amanz (datati tutti intorno alla metà del XII secolo) mostrano l'innesto del romanzo d'avventura sull'idillio; in particolare, senza che sia possibile stabilire un'interdipendenza fra le opere, la studiosa riconosce che elementi comuni ai tre testi sono la separazione della coppia a causa di differenze religiose o sociali (rispettivamente Floire et
} 
$n^{3}$ - risiede invece nel suo significato più profondo, che - in assenza di coordinate storiche plausibili e probabili e quindi di individuazione del pubblico cui era primariamente destinata l'opera - non si riesce a cogliere nitidamente, seppure l'intento parodico - come vedremo ${ }^{4}$ appare determinante e si definisce come sua cifra identificativa.

Quanto alla datazione la critica oscilla tra la fine del XII secolo e una generica iscrizione al XIII secolo ${ }^{5}$, anche se assai recentemente è stata avanzata la proposta di collocare la chantefable dopo il 1222 e di attribuirla a Jean Renart ${ }^{6}$.

È passato invece per lo più inosservato quanto rilevato da Brian Blakey in un'indagine compiuta nel 1968 sull'unico manoscritto che tramanda l'opera, oggi Bibliothèque nationale de France (Paris, BnF, fr. 2168: cc. 70r-80v) e risalente alla seconda metà del XIII secolo.

Infatti, già in passato la critica si era interrogata sulla frase del cap. XXIV, 4-5 ains li desronpent ses dras qu'a painnes peust on nouer desus el plus entier, poiché la lettura desus non era ritenuta sicura $^{7}$. In tale espressione Blakey ha pensato di trovare un riferimento alla pratica di trasportare denaro legato in un abito, come attestato da un passo dell'Huon de Bordeaux (vv. 4986-4988), e confermata anche altrove. E un controllo ai raggi ultravioletti del passo nel manoscritto ha rivelato la lettura de scu con un'errata divisione delle parole che si osserva anche in altri luoghi del manoscritto stesso, lettura che andrebbe interpretata $d$ 'escu con una costruzione partitiva di $d e$, riscontrabile anche altrove (traduzione: anzi gli strappano gli abiti così potrebbe difficilmente avere legato uno scudo nella parte più larga [dei suoi vestiti]). La menzione dell'escu rinvierebbe all'epoca di Luigi IX, precisamente agli anni 1266 e 1270 quando essa

Blanchefleur e Aucassin et Nicolette) o di rivalità tra famiglie (Piramus et Thisbé), e il fatto che i protagonisti si conoscono e si amano dall'età più tenera. Per ultimo Friedrich Wolfzettel, «Le paradis retrouvé : pour une typologie du roman idyllique», in Le Récit idyllique, op. cit., pp. 59-77, parla di ripresa dei dati iniziali di Floire et Blanchefleur in Aucassin et Nicolette, con una riduzione tuttavia della simbolica paradisiaca propria a quel testo.

${ }^{3}$ Così ad esempio si esprime Claude Roussel, «Mots d'emprunt et jeux de dupes dans Aucassin et Nicolette», Romania, 117 (1999), pp. 423-447, spec. p. 423.

${ }^{4}$ Cfr. infra.

${ }^{5}$ L'oscillazione temporale tra fine del XII secolo e inizi del Duecento è stata sostenuta da Edelgard Du Bruck, «The Audience of Aucassin et Nicolete: Confidant, Accomplice and Judge of its Author», Michigan Academician, 5 (1972), pp. 193-201, spec. pp. 194 e 199, che tuttavia sembra contraddirsi quando fa di Luigi IX di Francia la figura cui autore e pubblico possono aver pensato in relazione alla tolleranza di Aucassin verso Bougars e alla sua generosità nei confronti del bovaro. Per ultima, cfr. Sarah Kay, «Genre, parody, and spectacle in Aucassin et Nicolette and other short comic tales», in Simon Gaunt e Sarah Kay (edd.), The Cambridge Companion to Medieval French Literature, Cambridge, University Press, 2008, pp. 167-180, spec. p. 167

${ }^{6}$ Simonetta Mazzoni Peruzzi, «Du nouveau sur Aucassin et Nicolette», in Jean Lecointe, Catherine Magnien, Isabelle Pantin e Marie-Claire Thomine (edd.), Devis d'amitié. Mélanges en l'honneur de Nicole Cazauran, Paris, Champion, 2002, pp. 39-69.

${ }^{7}$ Maria Antonia Liborio, Aucassin et Nicolette, ed. cit., seguendo l'edizione di Roques, cit., edita desu. 
comparve per la prima volta, e quindi Aucassin et Nicolette andrebbe datato almeno alla seconda metà inoltrata del XIII secolo ${ }^{8}$.

Quanto invece alla composizione sociale del pubblico cui si rivolgeva l'anonimo della chantefable, un'indagine codicologica recentissima e dettagliata ha restituito l'idea alla base della costituzione del manoscritto stesso.

Infatti, la collezione apparentemente eterogenea, fatta di lais e fabliaux, di testi storici e moralizzanti, entro cui è incastonato Aucassin et Nicolette, appare in realtà dominata da due generi fondamentali della narrativa breve, i lais e i fabliaux, posti - non a caso - in apertura e in chiusura. La chantefable si pone proprio a metà strada tra la matière de Bretagne, cui rinviano i lais di Marie de France e quelli anonimi e l'Atre périlleux, e la materia favolistica rappresentata dalle Fables della stessa Marie e i fabliaux, e costituisce quindi una sorta di testo cerniera che presiede alle dinamiche intertestuali del codice tra la scrittura idillica e quella satirica9 ${ }^{9}$.

Così se la vicenda d'amore dei due protagonisti si svela nella parodia dei testi che precedono nel manoscritto e che rinviano alla letteratura romanzesca (cui ascrivere anche i lais), invece il discorso blasfemo di Aucassin sull'Inferno, quasi in apertura al cap. VI, prepara e permette la connessione con il primo testo che segue nel manoscritto la chantefable, ossia le Songe d'Enfer di Raoul d'Houdenc, mentre la battaglia parodica ai capp. VIII-X di Aucassin et Nicolette (cui andrebbe aggiunta quella di Torelore, capp. XXX-XXXII) anticipa La Bataille de Caresme et de Charnage e le fabliau Sacristain, ossia i due testi che a loro volta sono stati copiati dopo le Songe d'Enfer ${ }^{10}$.

La prevalenza di testi di narrativa breve, oltre all'evidente coloritura piccarda del manoscritto, sembrano rinviare all'ambiente urbano della Piccardia, che in pieno XIII secolo, e ancor più propriamente nella seconda metà, mostra di aver pienamente acquisito un ruolo di prestigio economico e di saper godere delle produzione letteraria anche cortese, spesso adattata alle nuove esigenze, per cui è a questo pubblico, di uomini e donne borghesi che si rivolge l'anonimo con riferimenti espliciti (si que vous avés oï et entendu) chiedendo la sua complicità e al tempo stesso mirando al suo divertimento.

L'analisi che segue, fondata sull'analisi del cap. XI della chantefable, se ha consentito da un lato di individuare in esso un complesso intreccio letterario posto sotto il segno della pastorella, e in particolare dell'esemplare antesignano del genere, dall'altro di confermare

\footnotetext{
${ }^{8}$ Brian Blakey, «Aucassin et Nicolette, XXIV, 4», French Studies, 22 (1968), pp. 97-98.

${ }^{9}$ Serena Lunardi, «Lire les fabliaux au Moyen Âge et au XVIII ${ }^{\mathrm{e}}$ siècle : les manuscrits Paris, BNF, fr. 2168 et Paris, Arsenal, 2770», Études françaises, 48 (2012), pp. 59-93, spec. pp. 70-84.

${ }^{10}$ Keith Busby, «Le contexte manuscrit du Songe d'Enfer de Raoul de Houdenc», in Yasmina Foehrr-Janssens e Olivier Collet (dir.), Le recueil au Moyen Âge : le Moyen Âge central, Turnhout, Brepols, 2010, pp. 47-61, spec. p. 51.
} 
l'ipotesi di Blakey circa la datazione avanzata dell'opera con delle inedite connessioni con la produzione teatrale arrageoise.

\section{LA STRUTTURA DI AUCASSIN ET NICOLETTE E IL SUO RAPPORTO CON LA SCRITTURA TEATRALE}

Come già detto, l'originalissima struttura formale rende Aucassin et Nicolette un'opera unica, trattandosi dell'unico esempio del genere cui l'autore ascrive il suo scritto, la chantefable appunto, termine che non a caso è un hapax ${ }^{11}$, dando prova in tal modo di uno sperimentalismo ardito.

In effetti, nel testo si alternano in maniera mirabile per solidità e regolarità, lasse di eptasillabi assonanzati con il petit vers finale di 4 o 5 sillabe a mo' di refrain, destinati a essere cantati come rivela la trasmissione della melodia, e parti in prosa destinate invece a essere recitate, per cui le lasse appaiono come la conclusione di quanto appena detto nella sezione in prosa precedente e il punto di partenza di quella che segue, secondo un particolare meccanismo di incatenamento con rare eccezioni ${ }^{12}$. Le lasse allora non costituiscono una sorta di pausa lirica, piuttosto esse informano e deformano, e la stretta comunione tra le diverse parti in versi e in prosa obbliga il pubblico a seguire con attenzione per non perdere elementi costitutivi nello sviluppo della vicenda ${ }^{13}$.

La particolare struttura così come le indicazioni che precedono ogni capitolo, or se chante prima delle inserzioni liriche, or dient et content et fablent prima delle sezioni in prosa (ove la pluralità delle azioni indicate per la prosa danno conto dei monologhi, dialoghi, descrizioni, invocazioni, espressione dei sentimenti $)^{14}$ inducono a ritenere che la chantefable venisse rappresentata.

Sull'origine di tale struttura si è a lungo indagato per individuare modelli anche latini e/o mediolatini (dalla satira menippea a Boezio $)^{15}$ e risulta piuttosto condivisibile quanto sottolineato dagli studiosi Hunt e Carmona ${ }^{16}$, i quali riconducono Aucassin et Nicolette nell'ambito di una tradizione letteraria già praticata nel Roman de la Violette da Gerbert de Montreuil e nel Guillaume de Dole da Jean Renart con l'inserimento di liriche preesistenti. La differenza

\footnotetext{
${ }^{11}$ Claude Roussel, «Mots d'emprunt...», art. cit., p. 423.

${ }^{12}$ Kaspar Rogger, «Étude descriptive...», art. cit., pp. 417 e 439.

13 Jean Trotin, «Vers et prose dans Aucassin et Nicolette», Romania, 97 (1976), pp. 481-508.

${ }^{14}$ Wlad Godzich and Jeffrey Kittay, The Emergence of Prose. An Essay in Prosaic, Minneapolis, University of Minnesota Press, 1987, pp. 80-81.

${ }^{15}$ John R. Reinhard, «The literary Background of the Chantefable», Speculum, 1 (1926), pp. 157-169; e Tony Hunt, «Precursors and Progenitors of Aucassin et Nicolette», Studies in Philology, 74 (1977), pp. 1-19, spec. 1-14, che si mostra critico rispetto a Reinhard.

${ }^{16}$ Rispettivamente Tony Hunt, «Precursors and Progenitors...», art. cit., pp. 14-19; e Fernando Carmona, El Roman lírico medieval, Barcelona, Promociones y Publicaciones Universitaires, 1988, pp. 227-275.
} 
sostanziale rispetto a tali opere che esalta l'unicità della chantefable e la rende un'antesignana, è che qui invece le sezioni liriche sono inventate ex novo e sono essenziali allo svolgimento della narrazione. D'altra parte la struttura di Aucassin et Nicolette può essere accostata al primo testo drammatico della letteratura francese come le Jeu de Robin et Marion, prodotto anch'esso in zona piccarda, in cui si riscontra alternanza di dialoghi, versi e musica ${ }^{17}$.

La commistione di strutture ascrivibili a più generi, la lassa all' epica, la prosa al romanzo del XIII secolo, l'eptasillabo alla tradizione lirica sia cortese sia popolareggiante, fornisce quindi un'indicazione inequivocabile circa il contenuto dell'opera in cui vengono ripresi e reinterpretati, come vedremo, motivi e generi letterari diversi; se poi la scelta dell'eptasillabo andasse interpretato come parodia dell'ottosillabo proprio ai romanzi cortesi come ammesso da $\operatorname{Harden}^{18}$, si troverebbe già nella struttura una risposta alla questione fondamentale che concerne Aucassin et Nicolette circa l'intento compositivo dell'anonimo.

\section{LA PARODIA COME INTENTO COMPOSITIVO DELLA CHANTEFABLE}

Infatti, ad una interpretazione inaugurata nel 1950 da Pauphilet ${ }^{19}$ e poi fatta propria da altri critici - secondo cui Aucassin et Nicolette mira a fare la parodia di diversi generi letterari (e a questa nozione Jodogne dieci anni dopo ha aggiunto anche quella di pastiche $)^{20}$ sono seguite tra il 1969 e il 1979 quelle di Ménard e soprattutto di Hunt i quali hanno negato decisamente la lettura parodica, perché a parere del primo l'autore avrebbe dovuto sottolineare il suo intento e seguire più da vicino i suoi modelli ${ }^{21}$; d'altra parte il suo pubblico - che Hunt presume fosse di modesta cultura -, per saper cogliere la parodia, avrebbe dovuto possedere uno spiccato spirito critico, con una coscienza letteraria sviluppata e una raffinata sensibilità verso le allusioni presenti nella chantefable $e^{22}$.

La posizione decisa di Hunt che rivendicava per la parodia non tanto una ripresa di testi, motivi o generi, quanto di stile, ha obbligato i critici a interrogarsi su di esso, come già aveva fatto Vance

\footnotetext{
${ }^{17}$ Robert Harden, «Aucassin et Nicolette as Parody», Studies in Philology, 63 (1966), pp. $1-9$, spec. p. 8.

${ }^{18}$ Ibid., p. 3.

${ }^{19}$ Albert Pauphilet, Les legs du Moyen Age. Études de littérature médiévale, Melun, Librairie d'Argences, 1950, pp. 239-248.

${ }^{20}$ Omer Jodogne, «La parodie et le pastiche dans Aucassin et Nicolette», Cahiers de l'Association internationale des études françaises, 12 (1960), pp. 53-65.

${ }^{21}$ Philippe Ménard, Le rire et le sourire dans les romans courtois en France au Moyen Age (1150-1250), Genève, Droz, 1969, pp. 518-521; ma cfr. anche Id., «La composition d'Aucassin et Nicolette», in Mélanges de philologie et de littérature romanes offertes à Jeanne WatheletWillem, Liège, Association des Romanistes de l'Université, 1978, pp. 413-432.

${ }^{22}$ Tony Hunt, «La parodie médiévale: le cas d'Aucassin et Nicolette», Romania, 100 (1979), pp. 341-381.
} 
che aveva proposto di vedere in Aucassin et Nicolette una commedia del linguaggio, con la giustapposizione di stili letterari differenti e incompatibili, i quali vengono definiti solo attraverso il contrasto: così ricorrendo all'alternanza tra prosa e poesia, l'anonimo coglie nel linguaggio letterario una molteplicità di funzioni, e testa il valore dei personaggi che derivano la loro natura dalla poesia, innestando le loro avventure nel discorso in prosa ${ }^{23}$. Da parte sua Smith, proprio nello stesso anno dell'articolo di Hunt, aveva definito il testo come una commedia stilistica in relazione al modo espressivo, per cui emerge un contrasto tra un genere rappresentato e lo stile atteso, ma poi non rispettato ${ }^{24}$.

L'interpretazione parodica tuttavia ha ripreso quota grazie a un'attenta disamina delle posizioni critiche attraverso l'analisi di episodi significativi della chantefable ${ }^{25}$, e ad una più approfondita indagine sul lavoro dell'anonimo con la sua volontà di sconcertare e disorientare continuamente il pubblico attraverso il ricorso a tecniche innovative - come ad altre già collaudate - con un effetto di patchwork deliberato ${ }^{26}$; ma soprattutto grazie ad una definizione più elastica di parodia non limitata a un solo testo con volontà dispregiativa, e la contestuale valorizzazione dell'intertestualità che risulta consustanziale alla lettura parodica stessa ${ }^{27}$.

L'anonimo avrebbe quindi giocato a mettere in scena le convenzioni letterarie del mondo della cortesia, quelle dell'epica e del romanzo, ma anche quelle riconducibili alla lirica e all'agiografia e quindi al fabliau $^{28}$, per evidenziarne la loro inflessibilità attraverso

${ }^{23}$ Eugene Vance, «The Word at Heart: Aucassin et Nicolette as a Medieval Comedy of Language», Yale French Studies, 45 (1970), pp. 33-51.

${ }^{24}$ Nathaniel B. Smith, «Aucassin et Nicolette as Stylistic Comedy», Kentucky Romance Quarterly, 26 (1979), pp. 479-490.

${ }^{25}$ Claude Roussel, «Mots d'emprunt...», art. cit.

${ }^{26}$ Jill Tattersall, «Shifting Perspectives and the Illusion of Reality in Aucassin et Nicolette», French Studies, 38 (1984), pp. 257-267. La definizione è a p. 259.

${ }^{27}$ Claude Roussel, «Mots d'emprunt...», art. cit., p. 427.

${ }^{28} \mathrm{Su}$ tutti, si vedano al riguardo le osservazioni di June Hall Martin, Love's fools: Aucassin. Troilus, Calisto and the Parody of the courtly Lover, London, Tamesis Books Limited, 1972, pp. 23-36, per cui Aucassin sembra comportarsi come Lancelot quando è dimentico di sé, e d'altra parte l'apparizione di un bovaro mentre rinvia al genere della pastorella ove è rappresentato il mondo rustico, ha come modello diretto l'incontro di Calogrenant con un uomo mostruoso ne Le Chevalier au lion di Chrétien de Troyes; invece la costruzione della loge e la trasformazione di Nicolette in giullare alludono alla vicenda tristaniana declinata pure da Marie de France in Chevrefeuil. Un riepilogo complessivo dei vari testi o generi parodiati in Aucassin et Nicolette, così come individuati da vari studiosi, si può leggere in Rudy S. Spraycar, «Genre and Convention in Aucassin et Nicolette», Romanic Review, 76 (1985), pp. 94-115, da cui emergono interessanti connessioni con la Prise d'Orange e il Roman de Renart; appartengono invece a Spraycar quelle istituite con la Chanson de Roland per il riferimento al guado intorbidato nell'episodio di Torelore, e i testi agiografici per il particolare della bianchezza dei piedi di Nicolette. Anche Ramón García Pradas, «Aucassin et Nicolette: reencuentro y parodia del cantar de gesta y la narrativa cortés», in Flor M. ${ }^{a}$ Bango de la Campa, Antonio Niembro Prieto e Emma Álvarez Prendes (edd.), Intertexto y polifonía. Homenaje a M. ${ }^{a}$ Aurora Aragón, 2 vols., Oviedo, Universidad de Oviedo, 2008, vol. I, pp. 273-281, rileva nella chantefable la critica ironica dell'eroe e del genere epici, e in particolare della Chanson de Roland: infatti, 
una serie di meccanismi quali le ripetizioni, l'esagerazione, l'inversione, e ancora la mancanza di accuratezza, l'evocazione della realtà, infine la decontestualizzazione delle formule, ciò che nel suo insieme produce un effetto di incongruità e quindi l'humour. Non solo, ma il gioco sulle convenzioni appare assai più complesso ed elaborato, poiché mentre nella prima parte della chantefable le convenzioni non sono seguite come ci si potrebbe aspettare, nella seconda parte quando ormai ci si è abituati al rovesciamento, esse vengono invece sorprendentemente rispettate ${ }^{29}$.

In tal senso allora il prologo assume tutta la sua importanza per la decifrazione della chantefable: in esso infatti Nicolette - menzionata per prima - è presentata come oggetto d'amore, Aucassin invece sembra un personaggio attivo capace di prouesces, quando poi lo sviluppo della narrazione rivelerà esattamente il contrario nell'episodio di Bougars e in quello di Torelore ${ }^{30}$, seppure nello stesso reame di Torelore con la rappresentazione del monde à l'envers i ruoli dei due protagonisti sembrino aderire al canone.

Se quindi l'intento dell'anonimo è quello di rivelare i difetti della tradizione cortese e di svalutarla, rendendo le convenzioni trasparenti e oggetto di parodia, appare senz'altro più calzante la datazione dell'opera almeno al pieno Duecento. E tuttavia, piuttosto che pensare solo alla classe medio-bassa come vuole Du Bruck ${ }^{31}$, e al pubblico di laici non coltissimo ma desideroso di imparare come ammesso da Lunardi in relazione al manoscritto ${ }^{32}$, bisognerà allargare l'uditorio della chantefable e ammettere anche un pubblico colto, di scuola in grado di apprezzare la parodia della letteratura seria cortese, quello stesso cui si rivolgevano i trovieri dei puys letterari di cui l'anonimo faceva verosimilmente parte, e che egli apostrofa in qualità di roi del siecle nel discorso sul Paradiso e sull'Inferno del cap. VI ${ }^{33}$.

mentre Rolando non si lamenta di vedersi separato da Alda, Aucassin piange per l'assenza di Nicolette; inoltre all'inizio del racconto egli accetta di combattere solo in base al ricatto e alla menzogna del padre, e la stessa scena dell'equipaggiamento con le armi - ripetuta nei capp. VIII e IX, secondo la tecnica delle lasse similari - viene ridimensionata rispetto al cliché epico, perché i pensieri di Aucassin sono tutti rivolti alla sua amata. Infine Eugene Vance, Mervelous Signals. Poetics and Sign Theory in the Middle Ages, Lincoln-London, University of Nebraska Press, 1986, spec. pp. 152-183 oltre a sottolineare il ruolo della pastorella medievale nella chantefable (per cui cfr. infra), ricorda la tradizione agiografica nell'episodio del ferimento di Aucassin nella foresta e quindi il fabliau per l'episodio del regno di Torelore.

${ }^{29}$ Anne Elizabeth Cobby, Ambivalent Conventions. Formula and Parody in Old French, Amsterdam-Atlanta, Rodopi, 1995, pp. 55-81.

${ }^{30}$ Così sottolinea Kevin Brownlee, «Discours as Proueces in Aucassin et Nicolette», Yale French Studies, 70 (1986), pp. 167-182.

${ }^{31}$ Edelgard Du Bruck, «The Audience of Aucassin et Nicolete...», art. cit., p. 194.

${ }^{32}$ Serena Lunardi, «Lire les fabliaux...», art. cit., p. 82.

${ }^{33}$ Mariantonia Liborio, «Aucassin et Nicolette: i limiti di una parodia», Cultura Neolatina, 30 (1970), pp. 156-171, spec. pp. 162-163; ma si veda anche Tony Hunt, «La parodie médiévale...», art. cit., pp. 351-352 che ridimensiona i riferimenti di Liborio ai clercs presenti alle riunioni del Puy e a una retorica scolastica. 


\section{IMPLICAZIONI IDEOLOGICHE E ATTENZIONE ALLA REALTÀ PROVENZALE}

La parodia come cifra stilistica e contenutistica di Aucassin et Nicolette non determina solo la destrutturazione della tradizione letteraria, ma consente anche il rifiuto di qualsiasi ideologia precostituita e di scrittura a tesi, attraverso la rappresentazione dello scarto e dell'alterità ${ }^{34}$, e quindi del relativismo culturale incarnato proprio dai due protagonisti ${ }^{35}$ : essi si amano nonostante le apparenti differenze sociali (solo alla fine si scoprirà che Nicolette è figlia del re arabo di Cartagena) e soprattutto le loro differenti origini, anch'esse interpretate secondo il ben collaudato sistema di inversione, come rivelano i loro nomi. Infatti Aucassin è cristiano ma porta il nome arabo Al Kasim del re di Cordova tra il 1019 e il 1021; lei invece saracena poi battezzata, ha un nome cristiano che rinvia a San Nicola ${ }^{36}$.

Se allora l'ignoranza iniziale sulle origini di Nicolette e il disvelamento finale pongono la 'questione araba' e appaiono come metafora per il dialogo e la differenza ${ }^{37}$, tuttavia lo svolgimento della vicenda tra $i$ due protagonisti non farebbe altro che indicare la tolleranza dell'alterità come condizione primaria dell'essere e della scrittura, ma anche del piacere e della conoscenza tra due mondi ${ }^{38}$.

Tuttavia dietro i nomi dei protagonisti potrebbero celarsi anche altri riferimenti secondo la tecnica collaudata del pastiche. Infatti la presenza nella chantefable di motivi riconducibili al mondo provenzale a partire dalla geografia - la vicenda infatti prende il via a Beaucaire sul Rodano e lì si conclude ${ }^{39}$ - ha spinto Dufournet a ulteriormente indagare se dietro i nomi dei due protagonisti si possano intravedere anche altri significati compatibili con tale contesto: così Aucassin sarebbe un diminutivo da aucassa, a sua volta derivato da auca, quindi 'oca', animale densamente connotato; mentre Nicolette discenderebbe da nicola, anch'esso sentito come diminutivo

\footnotetext{
${ }^{34}$ Claudio Galderisi, «Aucassin et Nicolette. La rencontre heureuse de deux épistémè», in Sophie Basch, André Guyaux e Gilberts Salmon (dir.), Orients littéraires. Mélanges offertes à Jacques Huré, Paris, Champion, 2004, pp. 153-164.

${ }^{35}$ María Rosa Menocal, «Signs of the Times : Self, Other and History in Aucassin et Nicolette», The Romanic Review, 80 (1989), pp. 497-511.

${ }^{36}$ Patrice Uhl, «Le Royaume de Torelore dans Aucassin et Nicolette: entre utopie et topique», in Marie-Françoise Bosquet, Serge Meitinger e Bernard Terramorsi (dir.), Aux confins de l'ailleurs: voyage, altérité, utopie. Hommages offerts au Professeur Jean-Michel Racault, Paris-Saint-Dénis, Klincksieck-Université de la Réunion, 2008, pp. 207-220, spec. pp. 212213. Tuttavia Robert Griffin, "Aucassin et Nicolette and the Albigensian Crusade», Modern Language Quarterly, 26 (1965), pp. 243-256, spec. a p. 247 ha segnalato pure il poeta arabo Al-Kâsim del XII secolo, come d'altra parte aveva già proposto Hugo Brunner, Über 'Aucassin und Nicolete', Halle, s.n, 1880, p. 12.

${ }^{37}$ María Rosa Menocal, «Signs of the Times...», art. cit., p. 508.

${ }^{38}$ Claudio Galderisi, «Aucassin et Nicolette...», art. cit., p. 164.

${ }^{39}$ María Rosa Menocal, «Signs of the Times...», art. cit., p. 503; e Patrice Uhl, «Le Royaume de Torelore...», art. cit., pp. 212-214, che ricorda come al tempo di Lacurne de SaintePalaye, autore di una traduzione della chantefable nel 1752, Aigues-Mortes fosse definita Pays de turelure.
} 
dell'occitanico nica, e usato in espressioni popolari con il significato di faire la nique, quindi 'fare lo sberleffo a qualcuno' ${ }^{40}$.

D'altra parte al mondo meridionale e in particolare alla crociata contro gli albigesi sembra rinviare secondo Griffin il riferimento a Beaucaire che fu assediata nel maggio 1216 da Simon de Montfort con 10.000 dei suoi uomini, lo stesso numero degli assedianti di Beaucaire in Aucassin et Nicolette. Anche le personalità del conte Garin e di suo figlio Aucassin, come le loro azioni, potrebbero essere ispirate a quelle di Raimondo VI, protettore del catarismo, e di suo figlio Raimondo VII ${ }^{41}$.

\section{LA RIPRESA PARODICA DI MOTIVI E MODALITÀ PROPRI ALLA LIRICA}

Proprio quest'apertura al mondo provenzale obbliga a riconsiderare con più attenzione la ripresa parodica - così insistita nella chantefable - di motivi e modalità proprî al discorso lirico di entrambe le tradizioni letterarie galloromanze, ripresa già segnalata dalla critica ma negata frettolosamente da Ménard ${ }^{42}$ e poi ridimensionata da Carmona, che attribuisce alle sezioni in versi solo una funzione lirica ${ }^{43}$.

In effetti il lamento di Nicolette rinchiusa nella torre al cap. V sembra evocare le chansons de toile o le canzoni di malmaritata, benché esso si concluda con il deciso proponimento della protagonista di uscire di prigione se le si offrirà l'occasione ${ }^{44}$. Invece il canto di Aucassin all'estoilete al cap. XXV è stato identificato con una sotte-chanson, e tale definizione si può accogliere solo nel senso di parodia del grand chant intonato dall'amante ${ }^{45}$.

Quanto all'alba, i motivi che la connotano sono ripresi e distribuiti lungo i capitoli XII-XVI tra sezioni in versi e sezioni in prosa, e anche in questo caso si osserva la parodia dei caratteri comuni al genere e il rovesciamento dei ruoli. Infatti Aucassin e Nicolette non hanno passato la notte insieme, non discutono circa il piacere derivante dal loro amore, piuttosto sono dubbiosi del loro sentimento, non si scambiano promesse circa il loro futuro, infine è Nicolette ad andarsene senza mostrare riluttanza, mentre Aucassin esprime rimpianto e piange; lo stesso canto della gaite che si legge nel cap. XV e che di solito si rivolgeva all'uomo, inizia invece con un elogio di

${ }^{40}$ Aucassin et Nicolette, Jean Dufournet (ed.), Paris, Flammarion, 1973, p. 12.

${ }^{41}$ Robert Griffin, «Aucassin et Nicolette...», art. cit., aggiunge al riguardo anche altri elementi che rinvierebbero al catarismo, come la preferenza di Aucassin per l'Inferno (cap. VI), e il riferimento al rogo (capp. VI e XVI); d'altra parte egli osserva come i Valdesi, alleati degli Albigesi, fossero assai numerosi in Piccardia.

${ }^{42}$ Philippe Ménard, Le rire et le sourire..., op. cit., pp. 518-519.

${ }^{43}$ Fernando Carmona, El Roman lírico..., op. cit., p. 238.

${ }^{44}$ Mariantonia Liborio, «Aucassin et Nicolette...», art. cit., nota 21 a p. 159 e p. 161

${ }^{45}$ Omer Jodogne, «La parodie et le pastiche...», art. cit., p. 65. 
Nicolette secondo lo stile cortese, ma si conclude con un avvertimento alla fanciulla in modo non letterario, quasi fosse un telegramma ${ }^{46}$.

Anche altri motivi della chantefable sono stati ricondotti alla lirica di tradizione trobadorica, come il riferimento alla primavera e al canto degli uccelli nei capp. XX, XXVI e XXXIX ${ }^{47}$; e addirittura per Rea l'alternanza tra versi fatti di musica e prosa sarebbe da ricondurre alla pratica testuale delle vidas e delle razos, come assicurano le frasi formulari quali Aucassin fu de Biaucaire /d'un castel de bel repaire (cap. III) e le formule di transizione del tipo Quant Aucassins oï dire Nicolete que ... (cap. XIV), proprie a quel tipo di composizioni ${ }^{48}$.

\section{IL GENERE PASTORELLA}

Tuttavia, il genere lirico che appare più frequentemente evocato nella chantefable è la pastorella, e nonostante ciò essa non è stato oggetto sinora di uno studio specifico.

I pastori, cui va aggiunto anche il vilain dalle mostruose fattezze del cap. XXIV, hanno piena dignità di azione e di parola soprattutto nella chantefable. Essi entrano in scena nel cap. XVIII quando Nicolette in fuga li incontra e li prega di riferire ad Aucassin che essi ben conoscono, un messaggio metaforico: una bestia di gran valore, cioè se stessa, e capace di guarire dai mali lo stesso Aucassin, si nasconde nella foresta e il ragazzo deve andare a cacciarla; i pastori non le credono, ma dietro il pagamento di un compenso di cinque soldi accettano di fare da messaggeri. Finalmente al cap. XX Aucassin, spinto da un cavaliere, decide di raggiungere la foresta ove incontra i pastorelli che mangiano e si divertono tanto da cantare una canzoncina, nella quale alludono ai due protagonisti della chantefable (cap. XXI). Nel capitolo successivo Aucassin chiede ai pastorelli di ripetere la canzone, ma loro si rifiutano e solo dopo il pagamento di dieci soldi da parte di Aucassin, decidono di riferire il messaggio di Nicolette. Lasciati i pastori al cap. XXIII, poco più avanti egli incontra il vilain dalle orride fattezze e di cristianiana memoria, che dinanzi alle lamentele di Aucassin per aver perso il proprio levriero bianco - altra metafora per indicare Nicolette -, racconta la sua penosa storia di povertà e di sfortuna per aver perso uno dei suoi buoi. Aucassin decide allora di elargire una somma all'uomo, questa volta di venti soldi per il riscatto dell'animale (cap. XXIV).

${ }^{46} \mathrm{Su}$ tutti, si vedano Williams S. Woods, «The Aube in Aucassin et Nicolette», in John Mahoney e John Esten Keller (edd.), Mediaeval Studies in Honor of Urban Tigner Holmes, jr., Chapel Hill, The University of North Carolina Press, 1965, pp. 209-215; Omer Jodogne, «La parodie et le pastiche...», art. cit., p. 65; Eugene Vance, «The Word at Heart...», art. cit., pp. 47- 48; June Hall Martin, Love's fools..., op. cit., p. 31; infine Rudy S. Spraycar, «Genre and Convention...», art. cit., p. 108.

${ }^{47}$ Fernando Carmona, El Roman lírico..., op. cit., pp. 270-271,

${ }^{48}$ John A. Rea, «The Form of Aucassin et Nicolette», Romance Notes, 15 (1974), pp. 504-508. 
Il rovesciamento delle situazioni messe in scena dal genere è evidente. L'incontro di Nicolette con i pastori richiama la cosiddetta pastorella classica: qui però non si tratta di un cavaliere, bensì di una fanciulla che vagando per la foresta incontra i pastori ${ }^{49}$; non solo, ma è lei a usare un linguaggio metaforico con evidenti sottintesi erotici, ciò che di solito era riservato al cavaliere in cerca di avventura; è lei infine a elargire del denaro ai pastori, mentre di solito è la pastorella che viene invogliata dal cavaliere con doni di varia natura. Anche nell'incontro di Aucassin con i pastori si assiste al rovesciamento, questa volta della pastorella cosiddetta oggettiva: mentre di solito il cavaliere assiste ai giochi e ai canti dei pastori che vivono le loro storie d'amore con pastorelle o se ne lamentano, e dispongono già dei propri strumenti musicali come del cibo, qui Aucassin riconosce nel canto dei pastori un'allusione alla propria vicenda sentimentale, e d'altra parte essi ricordano come i pasticcini, i coltellini e gli strumenti musicali saranno acquistati con i soldi elargiti loro da Nicolette; infine mentre i pastori cantano spontaneamente e ripetono i loro canti, qui la ripetizione del messaggio avviene dietro l'esborso di denaro.

Inoltre, come è stato già osservato da Vance ${ }^{50}$, la parodia del mondo pastorale e del genere letterario connesso, emerge quando l'anonimo racconta come i pastori escano dalla città per andare nella foresta, rivelando quindi un'identità sociale distinta da quella letteraria, a indicare quanto quel mondo rappresentato in poesia sia fittizio. A conferma del disvelamento della finzione sopraggiunge il fatto che i pastori si rivelano creature economiche, guidate unicamente dal desiderio del profitto, nel discorso più ampio della parodia del codice del commercio rappresentata in più luoghi della chantefable ${ }^{51}$.

Non solo, ma il significativo cambio di codice espressivo, indicato solo qui, per cui i pastori - unici intermediari tra i due protagoni$\mathrm{sti}^{52}$ - si rifiutano di ripetere ad Aucassin la canzone, limitandosi piuttosto a narrare il messaggio, dà la misura della consapevolezza da parte dell'anonimo della base socio-economica dell'interdiscorsività della chantefable $e^{53}$, ma - si potrebbe aggiungere - anche dell'importanza del ruolo giocato in essa dal genere pastorella, che pure conosce - come gli altri generi lirici rappresentati - una sensibile e significativa riduzione verso la prosa.

Quanto all'episodio del vilain, che già nell'Yvain sembra essere una risposta dello champenois all'entrata in scena in poesia di personaggi appartenenti al mondo pastorale ${ }^{54}$, è possibile riconoscere

\footnotetext{
${ }^{49}$ Nathaniel B. Smith, «Aucassin et Nicolette...», art. cit., p. 480.

${ }^{50}$ Eugene Vance, Mervelous Signals..., op. cit., pp. 167-168.

${ }^{51}$ Così riconoscono Eugene Vance, ibid., pp. 170-171; ed anche Virginia M. Green, «Aucassin et Nicolette: the Economics of Desire», Neuphilologische Mitteilungen, 79 (1995), pp. 197-206.

${ }^{52}$ Lo osserva Philippe Ménard, «La composition d'Aucassin et Nicolette», art. cit., a p. 416.

${ }^{53}$ Eugene Vance, Mervelous Signals..., op. cit., p. 172.

${ }^{54}$ Lucilla Spetia, 'Le conte de Bretaigne sont si vain et plaisant'. Studi sull'Yvain e sul Jaufre, Soveria Mannelli, Rubbettino, 2012, p. 104, nota 220.
} 
anche qui un rovesciamento denso di implicazioni sottese: se nel romanzo l'incontro è giocato sul piano del meraviglioso, in Aucassin et Nicolette assistiamo invece al confronto sociale; inoltre, se nel romanzo egli sa dominare i propri buoi, qui invece ne ha incredibilmente perso uno ${ }^{55}$; se infine nel romanzo il vilain creava disorientamento in Calogrenant, qui invece riconforta con le sue parole Aucassin che piange per la perdita del suo levriero. La presenza del vilain appare quindi paradossale perché a dispetto del suo aspetto rude e della sua assai modesta condizione sociale, egli sa molto ben parlare, tanto da fare la morale ad Aucassin; così come altrettanto paradossale è la storia che racconta, di un bue perduto mentre stava lavorando ${ }^{56}$.

Ai motivi che rinviano alla pastorella, vanno pure aggiunti l'attenzione straordinaria riservata dall'anonimo alla scelta lessicale di nomi di strumenti musicali e di appellativi di pastori proprî al genere, oltre che di diminutivi nel cap. $\mathrm{XXI}^{57}$; quindi la stessa descrizione fisica di Nicolette grasse et mole (cap. XXXIII, 5) sembra rinviare a quella di Marion nel Jeu de Robin et Marion ${ }^{58}$, cui si potrebbero aggiungere i riferimenti alle mameletes dures (XII, 24) e al cateron de sa mamele (cap. XIV, 23), che ricordano quelle delle pastorelle francesi; per ultimo, addirittura il nome di Torelore rinvierebbe ai refrains onomatopeici come turuluta, tureleure, teirelire riscontrabili in diversi esempi del genere ${ }^{59}$.

\section{IL CAPITOLO XI}

Quanto sin qui detto autorizza - e anzi sollecita - a riconsiderare con maggiore attenzione il capitolo XI della chantefable, ove è attestata la parola tipica dell' incipit delle pastorelle e considerata a ragione la marca del genere, ossia l'autrier ${ }^{60}$, che attestata fin dalla

${ }^{55}$ L'osservazione è di Douglas D. R. Owen, «Chrétien, Fergus, Aucassin et Nicolette and the Comedy of Reversal», in Peter S. Noble e Linda M. Paterson (edd.), Chrétien de Troyes and the Troubadours. Essays in memory of the late Leslie Topsfield, Cambridge, St. Catherine's College, 1984, pp. 186-194, spec. p. 192.

${ }^{56}$ Claude Roussel, «Mots d'emprunt...», art. cit., pp. 435-438.

${ }^{57}$ Così sottolineano Mariantonia Liborio, «Aucassin et Nicolette...», art. cit., p. 168; Nathaniel B. Smith, «Aucassin et Nicolette...», art. cit., p. 480; infine Rudy S. Spraycar, «Genre and Convention...», art. cit., p. 105.

${ }^{58}$ Nathaniel B. Smith, «The Uncourtliness of Nicolette», in Raymond J. Cormier (ed.), Voices of Conscience. Essays on Medieval and Modern French Literature in Memory of James D. Powell and Rosemary Hodgins, Philadelphia, Temple University Press, 1977, pp. 169-182, spec. pp. 175-176.

${ }^{59}$ L'osservazione è stata fatta da diversi studiosi, Aucassin et Nicolette, Dufournet, ed. cit., p. 185; Claude Roussel, «Mots d'emprunt...», art. cit., p. 440; quindi Patrice Uhl, «Le Royaume de Torelore...», art. cit., pp. 217-218. Tuttavia già Albert, Les legs du Moyen Age..., op. cit., p. 244, aveva parlato di Torelore come di un refrain di canzone derisoria.

${ }^{60}$ Elizabeth Schulze-Busacker, «L'exorde de la pastourelle occitane», Cultura Neolatina, 38 (1978), pp. 223-232. 
prima pastorella di Marcabruno L'autrer jost'una sebissa ${ }^{61}$, ha la sua origine in realtà nella lirica dei vanti di Guglielmo IX, Ben vueill que sapchon li pluzor ${ }^{62}$ cui il trovatore guascone rinvia in modo ironico, poiché proprio in un l'autrer imprecisato il duca d'Aquitania aveva conosciuto una défaillance sessuale ${ }^{63}$.

Ora, nel cap. XI Aucassin chiuso in prigione dal proprio padre, incomincia a lamentarsi e dopo aver invocato Nicolette, flors de lis, pensa di vedere proprio lei (e il pubblico è invitato a fare altrettanto) che l'altro ieri grazie a un curioso spogliarello aveva guarito un pellegrino gravemente malato di esvertin, ossia di follia, per poi ricordare i bei momenti trascorsi con lei e quindi invocare la propria morte. Lo spettacolo ha l'apparenza di un miracolo, annunciato quindi come innocente, sebbene si svolga non in santuario, ma finisce in realtà in una fantasia sessuale ${ }^{64}$ :

CAP. XI

Quant or voit li quens Garins de son enfant Aucassin qu'il ne pora departir de Nicolete au cler vis, en une prison l'a mis en un celier sosterin qui fu fais de marbre bis. Quant or i vint Aucassins, dolans fu, ainc ne fu si ; a dementer si se prist si con vos porrés oïr : «Nicolete, flors de lis, douce amie o le cler vis plus es douce que roisins ne que soupe en maserin. L'autr'ier vi un pelerin nes estoit de Limosin, malades de l'esvertin si gisoit ens en un lit, mout par estoit entrepris, de grant mal amaladis tu passas devant son lit, si soulevas ton traïn et ton peliçon ermin, la cemisse de blanc lin

5 tant que ta ganbete vit : garis fu li pelerins et tos sains, ainc ne fu si ; si se leva de son lit, si rala en son païs sains et saus et tos garis. Doce amie, flors de lis, biax alers et biax venirs, biax jouers et biax bordirs, biax parlers et biax delis, dox baisiers et dox sentirs, nus ne vous poroit haïr. Por vos sui en prison mis en ce celier sousterin u je fac mout male fin ; or m'i convenra morir por vos, amie»

${ }^{61}$ Per l'edizione del testo, si rinvia a Simon Gaunt, Ruth Harvey, Linda Paterson (edd.), Marcabru. A Critical Edition, Cambridge, Brewer, 2000, pp. 375-387.

${ }^{62}$ La lirica si può leggere in Guglielmo IX d'Aquitania, Poesie, Nicolò Pasero (ed.), Modena, Mucchi, 1973, pp. 157-186.

${ }^{63}$ Lucilla Spetia, «Gatti rossi e gatti neri: un mistero felino alle origini della pastorella?», in Angelica Rieger (ed.), L'Occitanie invitée de l'Euregio. Liège 1981-Aix la Chapelle 2008: Bilan et perspectives. Actes du IX $X^{\mathrm{e}}$ Congrès International de l'Association Internationale d'Études Occitanes (Aix-la-Chapelle, 24-31 août 2008), 2 vols., Aachen, Shaker, 2011, vol. I, pp. 543-555.

${ }^{64}$ Sarah Kay, «Genre, parody...», art. cit., p. 169. 
Il capitolo è particolarmente interessante almeno per due ragioni. Infatti il v. 21 riproduce letteralmente il v. 12 del prologo (vv. 10-15: Nus hom n'est si esbahis, / tant dolans ni entrepris, /de grant mal amaladis / se il l'oit, ne soit garis / et de joie resbaudis, / tant par est douce), e tale ripresa che si sostanzia del ricorso alla figura etimologica $^{65}$, determina un nesso incontestabile tra l'effetto dello spogliarello sul pellegrino malato e quello dell'intera chantefable sull'uditorio $^{66}$, e soprattutto indica in modo chiaro la centralità e l'importanza della scena dello spogliarello di Nicolete nell'economia del testo.

Non solo. La scena è preceduta e seguita da versi (rispettivamente i vv. 12-15 e vv. 32-36) che inquadrano e connotano il passo, densi di allusioni erotiche. Infatti nei primi alla virtù del giglio che rinvia alla purezza e alla castità, segue un riferimento sessuale trasparente con l'indicazione del pane che si bagna nel vino ${ }^{67}$; d'altra parte i vv. 32-36 forniscono un catalogo di azioni e sensazioni che alludono al piacere sessuale, con il vocabolo bordirs che potrebbe rinviare alla forma bordel già col significato di 'maison de prostitution', e costituiscono il rovesciamento parodico con inversione dei termini dei vv. 12-16 del cap. VII (Nicolete, biax esters, / biax venir et biax alers, / biax deduis et biax parlers, / biax borders et biax jouers, / biax baisiers, biax acolers), tanto che per i due capitoli si è parlato a ragione di effetto di lasse similari proprio alle chansons de geste ${ }^{68}$.

Le considerazioni svolte da Vance e Smith risultano appropriate, sebbene sia da rilevare che il nesso logico tra la scena dello spogliarello di Nicolette e l'amore totale di Aucassin per lei tanto da desiderare la morte, appare debole; d'altra parte una certa spregiudicatezza di Nicolette sembra emergere nel cap. XIII, quando costei gli rivela che per amore di lui attraverserebbe il mare per andarsene in un'altra terra (vv. 13-14), per cui Aucassin dichiara nel capitolo successivo che si ucciderebbe se venisse a sapere che qualcuno si fosse impadronito di lei, l'avesse portata nel letto e l'avesse messa sotto (XIV, 6: si vos asoignenteroit), svelando la tradizionale presa di posizione maschile verso le donne e la loro sessualità, ma anche l'esagerazione comica dell'ideale di fedeltà proprio alla letteratura cortese ${ }^{69}$.

La curiosa scena ricordata da Aucassin al cap. XI ha ovviamente interessato $i$ critici che hanno sostanzialmente avanzato tre ipotesi interpretative. La prima è stata sostenuta da Rogger ${ }^{70}$ secondo cui ci si

${ }^{65}$ Come riconosciuto da Álvaro Galmés de Fuentes, Aucassin y Nicolette, Madrid, Gredos, 1998, p. 22, e p. 53, nota 41.

${ }^{66}$ Mariantonia Liborio, «Aucassin et Nicolette...», art. cit., nota 4 a p. 156 osserva come tale vanteria nel prologo risulti inedita.

${ }^{67}$ Eugene Vance, «The Word at Heart...», art. cit., p. 48.

${ }^{68}$ Nathaniel B. Smith, «The Uncourtliness of Nicolette...», art. cit., pp. 173-175; e Id., «Aucassin et Nicolette...», art. cit., p. 487. Cfr. pure supra, nota 28.

${ }^{69}$ Jill Tattersall, «Social Observation and Comment in Aucassin et Nicolette», Neuphilologische Mitteilungen, 86 (1985), pp. 551-565, spec. p. 558.

${ }^{70}$ Kaspar Rogger, «Étude descriptive...», 70 (1954), art. cit., pp. 3-5. 
troverebbe in presenza di un'allusione a canzoni folkloriche sparite che attribuivano virtù magiche e terapeutiche alle parti segrete della donna, mentre Spitzer ${ }^{71}$ ha associato la capacità divina di Nicolette a quella di una fata, cui fanno riferimento i pastori nei capp. XVIII e XXII, sebbene Micha avesse ridimensionato l'ipotesi di Rogger ed affermato che l'anonimo si era ispirato ad una comparazione banale, quella della bellezza come miglior medicina ${ }^{72}$.

La seconda interpretazione fatta propria da Roques e quindi da Ménard $^{73}$, fa dello spogliarello di Nicolette un movimento di coquetterie.

La terza ipotesi invece vedrebbe nel passo la parodia dei testi agiografici in cui veniva rappresentato il potere miracoloso di un santo $\mathrm{o}$ addirittura di Gesù Cristo ${ }^{74}$. Particolarmente significative sono le ipotesi di Du Bruck ${ }^{75}$, che ha ricondotto la scena satirica del miracolo insieme al rovesciamento dei ruoli di Inferno e Paradiso così come descritti nel cap. VI, alla critica dell'anonimo verso le posizioni della Chiesa; e di Williamson ${ }^{76}$ che connette il nome di Nicolette a quello di san Nicola, il quale - andrebbe aggiunto - è il santo protettore delle ragazze da marito (ma anche di quelle avviate alla prostituzione). Circa la plausibilità di tale ipotesi, va tuttavia ricordata l'annotazione di Micha che aveva sottolineato l'anomala situazione descritta nel cap. XI, per cui il pellegrino una volta guarito, non si reca al santuario che stava cercando, piuttosto torna a casa ${ }^{77}$.

Soltanto Hunt ha evocato la nozione della dama cortese che sa guarire i malati, richiamando il noto verso 25 Per son joi pot malaus sanar della lirica Molt jauzens, mi prenc en amar di Guglielmo IX ${ }^{78}$.

Ora, proprio l'evocazione del primo trovatore risulta decisamente appropriata, perché se si legge con attenzione il passo ci si rende conto che l'anonimo della chantefable sta rappresentando a suo modo, quindi parodiandola, la famosa canzone del gatto rosso Farai un vers, pos mi sonelh dal sapore di un fabliau, quando Guglielmo andandosene da solo in Alvernia, oltre il Limosino, travestito da

${ }^{71}$ Leo Spitzer, «Le vers 2 d'Aucassin et Nicolette et le sens de la chantefable», in Id., Romanische Literaturstudien 1936-1956, Tübingen, Niemeyer, 1959, pp. 49-59, spec. p. 54 (si tratta della ristampa dell'articolo apparso in Modern Philology 45, 1947-1948, pp. 8-14).

${ }^{72}$ Alexandre Micha, «En relisant Aucassin et Nicolette», Le Moyen Age, 65 (1959), pp. 279-292, spec. p. 287 (poi ristampato in Alexandre Micha, De la chanson de geste au roman. Études de littérature médiévale, Genève, Droz, 1976, pp. 465-478).

${ }^{73}$ Rispettivamente Mario Roques, «Compte rendu» all'articolo di Kaspar Rogger, «Étude descriptive...», 76 (1955), più volte citato, spec. p. 118; e Philippe Ménard, Le rire et le sourire..., op. cit., nota 39 alla p. 641, con un rinvio a Ovidio, Amores III, 2, 27.

${ }^{74} \mathrm{Al}$ riguardo si vedano Harden, «Aucassin et Nicolette», art. cit., p. 7 secondo cui si tratta di un motivo da fabliau; Nathaniel B. Smith, «The Uncourtliness of Nicolette...», art. cit., p. 173; Virginia M. Green, «Aucassin et Nicolette...», art. cit., nota 8 a p. 206.

${ }^{75}$ Edelgard Du Bruck, «The Audience of Aucassin et Nicolete ...», art. cit., pp. 198-199.

${ }^{76}$ Joan B. Williamson, «Naming as a Source of Irony in Aucassin et Nicolette», Studi francesi, 17 (1973), pp. 401-409, spec. pp. 405-406.

${ }^{77}$ Alexandre Micha, «En relisant Aucassin et Nicolette», art. cit., p. 287.

${ }^{78}$ Tony Hunt, «La parodie médiévale...», art. cit., p. 364. Per l'edizione del testo di Guglielmo IX, cfr. Guglielmo IX d'Aquitania, Poesie, ed. cit., pp. 213-240. 
pelerin, riusciva a portare a compimento un'avventura straordinaria, giacendo con due donne per ben 188 volte, segno di un'ossessione erotica senza pari ${ }^{79}$.

Ed allora il malato di esvertin della chantefable è proprio lui, e la sua guarigione si compie attraverso uno spogliarello apparentemente pudico di Nicolette, che si limita (o almeno così è detto) a mostrare la propria ganbete.

Non solo. Il riferimento agli abiti di pregio della ragazza, peliçon ermin e cemisse de blanc lin (cui va aggiunto un bliaut de drap de soie nel cap. XII, 12) - attestati pure nella lirica francese Volez vos que je vos chant (RS 318), come opportunamente segnalato da Scheludko ${ }^{80}$ - e che sembrano fare la parodia all' abbigliamento della tozeta marcabruniana de L'autrer jost'una sebissa (v. 5-7: chap'e gonela pellissa / viest, e camiza traslissa / sotlars e caussas de laina), così come l'incipit $l$ 'autrier già ricordato, incastonano la scena all'interno del genere pastorella.

La connessione tra la canzone del gatto rosso e la pastorella non è casuale, perché - com'è noto - il primo esempio del genere, la già menzionata L'autrer jost'una sebissa di Marcabruno, mette in scena una saggia pastorella che, nel respingere le avances sessuali sempre più insistenti ed esplicite del cavaliere da identificare proprio con Guglielmo - che si era fatto protagonista della straordinaria impresa erotica nel Limosino al punto di proporle l'accoppiamento bruto (lui di sopra, lei di sotto) -, gli rammentava a mo' di rampogna il principio di mezura, e faceva valere contro la foudatz di quello il proprio sen nell'idea che l'accoppiamento era possibile solo tra persone «compatibili naturaliter» ${ }^{81}$.

All'identificazione di Nicolette con la pastorella marcabruniana contribuisce un elemento fortemente significativo: Nicolette è una ragazza di origini arabe, e secondo la ricostruzione avanzata per prima da Maria Luisa Meneghetti l'aggettivo mestissa che connota la tozeta di Marcabruno, va tradotto con 'meticcia', ossia araba, che per Lazzerini sarebbe da identificare con la Sulamita, nigra sed formosa del Cantico dei Cantici; non solo ma sulla base di elementi lessicali presenti in L'autrer jost'una sebissa come pure nell'altra pastorella di Marcabruno L'autrier a l'issida d'abriu, è possibile ammettere che l'ideazione dei testi sia avvenuta in Spagna all'epoca del suo

${ }^{79}$ Guglielmo IX d'Aquitania, Poesie, ed. cit., pp. 113-155.

${ }^{80}$ Dimitri Scheludko, «Zur Entstehungsgeschichte von Aucassin und Nicolete», Zeitschrift für romanische Philologie, 42 (1922), pp. 458-490, spec. p. 486. Per l'edizione del testo, il rinvio è a Karl Bartsch (ed.), Altfranzösische Romanzen und Pastourellen, Leipzig, Vogel, 1870, pp. 23-24 (rist. anastatica Genève, Slatkine Reprints, 1973).

${ }^{81}$ A tal riguardo si vedano gli studi fondamentali di Nicolò Pasero, «Sulla collocazione socioletteraria della pastorela di Marcabruno», L’immagine riflessa, 4 (1980), pp. 347-364; e «Pastora contro cavaliere, Marcabruno contro Guglielmo IX. Fenomeni di intertestualità in L'autrer jost'una sebissa (BdT 293, 30)», Cultura Neolatina, 43 (1983), pp. 9-25. 
soggiorno tra il 1134 e il 1143 presso la corte di Alfonso VII di Castiglia e di León ${ }^{82}$.

Ce n'è abbastanza allora per riconoscere nel cap. XI della chantefable un complesso intreccio letterario fatto di densissime allusioni che erano certamente colte e apprezzate dal pubblico, e per ammettere che il gioco parodico rappresentato fosse esilarante.

Infatti, il senher malato di follia erotica, ossia il primo trovatore, viene guarito dall'araba Nicolette, che a differenza della pastorella marcabruniana, non solo non gli si oppone, ma anzi qualcosa gli conce$\mathrm{de}$, la visione della propria ganbete, se non qualcosa di più consistente a fronte delle allusioni nel testo alla possibilità di Nicolette di giacere con altri, sconfessando così l'intento moralistico di Marcabruno.

Ma soprattutto aver parodiato il débat a distanza tra Marcabruno e Guglielmo IX significa indicare in modo inequivocabile la cifra espressiva della chantefable, poiché i due testi occitanici sono già a loro volta fortemente parodici (quello del gatto rosso parodiava infatti le chansons de rencontre mediolatine) $)^{83}$, e al tempo stesso esaltare il potere taumaturgico della letteratura e la capacità salvifica della parola, poiché esse favoriscono la guarigione attraverso il ricorso all'ironia, per cui Aucassin et Nicolette si conferma come commedia del linguaggio letterario.

Il cap. XI non costituisce allora solo la parodia del genere 'pastorella', pure praticata in altri capitoli della chantefable e che si rivela perciò genere letterario particolarmente fecondo di sviluppi interpretativi, ma proprio del gioco letterario in sé definitosi agli albori delle letterature romanze attraverso i suoi testi fondanti, e perciò l'espressione de grant mal amaladis appare nel prologo che offre la chiave di lettura dell'intera chantefable.

\section{Un Richiamo intertestuale a LE JeU de la Feuillée di Adam DE LA HALle}

Non solo. L'indagine intertestuale compiuta a partire dal cap. XI, l'unica che consente di cogliere appieno il valore e il senso della parodia, si rivela fondamentale per confermare la datazione tarda proposta per Aucassin et Nicolette, e addirittura a meglio circoscriverla.

${ }^{82}$ Per tutto ciò, il rinvio è a Maria Luisa Meneghetti, «Una serrana per Marcabru?», in O cantar dos trobadors. Actas do Congreso celebrado en Santiago de Compostela (26-29 abril 1993), Santiago de Compostela, Xunta de Galicia, 1993, pp. 187-198; Lucia Lazzerini, Letteratura medievale in lingua d'oc, Modena, Mucchi, 2001, pp. 77-78; nuovamente Maria Luisa Meneghetti, «Marcabru e le origini iberiche della pastorella», in Anne Amend- Söchting, Kirsten Dickhaut, Walburga Hulk et al. (eds.), Das Schöne im Wirklichen-Das Wirkliche im Schönen. Festschrift für Dietmar Rieger zum 60. Geburstag, Heidelberg, Universitätverlag C. Winter, 2002, pp. 135-142; infine Lucilla Spetia, «Alle origini della pastorella, un genere popolare», Studi Mediolatini e Volgari, 56 (2010), pp. 167-216.

${ }^{83}$ Sulla questione si vedano Lucilla Spetia, «Alle origini della pastorella...», art. cit.; e Id., «Gatti rossi e gatti neri...», art. cit. 
Infatti, il controllo lessicale sul vocabolo esvertin ${ }^{84}$ così densamente allusivo, rivela il suo utilizzo solo in un altro testo letterario, più propriamente teatrale che proviene dalla stessa area della chantefable, ossia le Jeu de la Feuillée di Adam de la Halle ${ }^{85}$.

Com'è noto, esso costituisce accanto all'altro Jeu composto dallo stesso Adam una tappa fondamentale del teatro medievale in volgare: mentre il Jeu de Robin et Marion rappresenta la prima pastorale drammatica, le Jeu de la Feuillée è considerato il primo testo di teatro profano francese, o ancor meglio la prima commedia borghese, dal sapore particolare ${ }^{86}$.

Come suggerisce pure il committente che lo ha ispirato, la Confrérie des jongleurs et des bourgeoises d'Arras (detta Carité Nostre Dame des jogleors et des borgois) formatasi nella seconda metà del XII secolo, al più tardi nel 1194 secondo quanto ricostruito da Berger $^{87}$, esso offre uno spaccato della vita quotidiana di Arras. Stanno a rivelarlo alcuni dei personaggi in scena, il padre stesso di Adam, dame Douche forse appartenente alla famiglia di Andrieu Douche, e tre dei suoi amici, ossia Rikier prospero commerciante da identificare forse con Adam Aurri, Guillot le Petit citato nei Congés di Baude Fastoul, infine Hane le Merchier menzionato dallo stesso Baude tra gli arbalestrieri della città ${ }^{88}$. Tuttavia, la rappresentazione non è neutra, poiché la città viene dipinta come il regno della frode, del sopruso e dell'avarizia ${ }^{89}$, e il testo si svela essere un virulento pamphlet politico contro i patrizi che detengono il potere ${ }^{90}$.

Particolarmente interessante è il titolo dell'opera: infatti il jeu (che nel senso di opera teatrale, conosce la piena e definitiva consacrazione proprio ad Arras $)^{91}$ fa riferimento al fogliame che ricopriva la piccola cappella, sorta di torre di pietra a forma di piramide, ove erano custodite le reliquie della Vergine, patrona dei giullari da cui - secondo la tradizione - avevano ricevuto una candela miracolosa per guarire le mal des ardents ${ }^{92}$. Questa domus di foglie e fiori veniva rinnovata a maggio di ogni anno, creandosi in tal modo una sorta di convergenza tra il culto della Vergine e i riti del Calendimaggio ${ }^{93}$.

\footnotetext{
${ }^{84}$ Cfr. i dizionari TL, III, 1507; e Godefroy, III, 667-668, s. v.

${ }^{85}$ Per l'edizione, si è scelta di seguire quella curata da Rosanna Brusegan: Adam de la Halle, Teatro. La commedia di Robin e Marion. La Pergola, Rosanna Brusegan (ed.), Venezia, Marsilio, 2004.

${ }^{86}$ Adam de la Halle, Teatro, Brusegan (ed.), ed. cit., p. 9.

${ }^{87}$ Roger Berger, Littérature et société arrageoises au XIII siècle: les chansons et dits artésiens, Arras, Commission départementale des monuments historiques du Pas-de-Calais, 1981, p. 86.

${ }^{88}$ Così ricorda Jean Dufournet, Adam de la Halle à la recherche de lui-même ou Le jeu dramatique de la Feuillée. Paris, SEDES, 1974, pp. 232-234.

${ }^{89}$ Adam de la Halle, Teatro, Brusegan (ed.), ed. cit., p. 58.

${ }^{90}$ Jean Dufournet, Adam de la Halle..., ed. cit., p. 59.

${ }^{91}$ Così sottolinea proprio Brusegan in Adam de la Halle, Teatro, ed. cit., p. 58.

${ }^{92}$ Roger Berger, Littérature et société..., op . cit., p. 86.

${ }^{93}$ Adam de la Halle, Teatro, Brusegan (ed.), ed. cit., p. 53.
} 
Tuttavia, il vocabolo feuilliée può anche alludere alla pergola menzionata in alcune pastorelle come luogo di incontro tra il cavaliere e la pastora (L'autrier alloie juant, RS 346, vv. 5-7: Si con j'aloie pansant, / si vi pastoure ombroiant / sus une foillie) o di riunione di pastori e pastore (A la foillie a Doumartin, RS 1363, vv. 1-4: A lai foillie a Doumartin / a l'entree dou tens novel / s'asamblerent par un matin / pastorelles et pastorelz) $)^{94}$.

Non solo, ma come mostrato da Rousse ${ }^{95}$, feuillie poteva anche rinviare a una forma di follia, ampiamente rappresentata nel Jeu. Infatti nell'opera, che non conosce l'unità d'azione, si succedono più storie, quella di Adam che crede di lasciare Arras per Parigi; quelle di Riquier e di Douce, e di un folle e di suo padre; infine quella degli amori della fata Morgana.

Ora, proprio nell'ambito della prima storia, dopo che un medico giudica il padre di Adam affetto del mal di sant Lienart, ossia di avarizia e per antifrasi anche di impotenza, e la prostituta Dame Douce di essere incinta, entra in scena un monaco che annuncia in presenza di Walet il matto la visita di Saint'Acaire, noto per guarire dalla demenza definita appunto eswertin (vv. 322-337):

\section{Li MoINES}

Segneur, me sires sains Acaires

Vous est chi venus visiter;

Si l'aprochiés tout pour ourer,

Et si meche chascuns s'offrande, 325

Qu'il n'a saint desi en Irlande

Que si beles miracles fache;

Car l'anemi de l'ome encache

Par le saint miracle devin
Et si warist de l'esvertin

Communement et sos et sotes.

Souvent voi des plus ediotes

A Haspre no moustier venir

Qui sont haitié au departir,

Car li sains est de grant merite;

Et d'une abenguete petite

Vous poés bien faire du saint.

La coerenza del riferimento all'esvertin nel testo di Adam de la Halle, e all'opposto l'incongruità di quello stesso riferimento nella chantefable in contesti entrambi densamente allusivi all'eros, consente di cogliere nel cap. XI di Aucassin et Nicolette una ripresa divertente e divertita dell'episodio del Jeu de la Feuillée: la figura di Sant'Acaire viene sostituita da quella di Nicolette, una guaritrice sui generis, e alla parodia dei testi agiografici ove si ricordavano i miracoli dei santi già praticata da Adam, l'anonimo vi ha volutamente sovrapposto e intrecciato con abilità e spregiudicatezza straordinarie la parodia della pastorella e della lirica del gatto rosso - lirica cui probabilmente allude Adam quando rinvia a sant Lienart ricordato al

${ }^{94}$ I due testi sono stati editi da Jean-Claude Rivière (ed.), Pastourelles. 3 vols. Genève, Droz, 1974-1976, vol. I, rispettivamente alle pp. 151-153, e 119-121.

${ }^{95}$ Henri Roussel, «Notes sur la littérature arrageoise du XIII ${ }^{\mathrm{e}}$ siècle», Revue des Sciences Humaines, 87 (1957), pp. 249-286, spec. pp. 280-281. 
v. 18 di Farai un vers pos mi sonelh di Guglielmo IX - per amplificare a dismisura il gioco intertestuale.

Non è un caso allora che l'autore di Aucassin et Nicolette abbia menzionato nel prologo la malattia da cui si può guarire, ossia la follia del pellegrino, in ciò imitando e parodiando proprio il Jeu de la Feuilliée, ove la stessa malattia e la sua guarigione sono ricordate nel prologo stesso (v. 8: Aprés grant maladie ensieut bien grans santés).

Non solo, il Jeu può aver fornito all'anonimo della chantefable anche l'idea del conflitto di Aucassin con il padre Garin, poiché il motore della commedia è costituito proprio dal conflitto di Adam con la moglie, e soprattutto con il padre, contento che il figlio riprenda gli studi a Parigi e così smetta di perdere tempo con una donna, ma deciso per avarizia a non voler sborsare un soldo per lui.

Infine, anche l'ispirazione del testo teatrale sembra trovare eco nella chantefable: infatti se il processo di critica della cortesia appare in modo giocoso nel Jeu de Robin et Marion, esso risulta ben più radicale proprio nel Jeu de la Feuillée, ove Adam de la Halle rappresenta il tramonto del sogno utopico del mondo ideale della cortesia, e in particolare dell'amore, di fronte alla drammaticità e alla bassezza di quello reale ${ }^{96}$, ma lo stesso disincanto dinanzi alla civiltà cortese ormai in dissolvimento connota senza dubbio anche la scrittura parodica di Aucassin et Nicolette.

Se allora anche il Jeu de la Feuillée rappresenta una fonte di ispirazione di Aucassin et Nicolette, tale connessione permette di proporre un termine post quem per la sua composizione.

\section{UNA NUOVA IPOTESI DI DATAZIONE DELLA CHANTEFABLE}

Com'è noto, il testo teatrale è datato al 1276 sulla base di elementi interni al testo stesso.

Innanzitutto, ai vv. 462-463 si fa riferimento alla morte recente di un papa che si era pronunciato contro i clercs bigami, di cui si parla nel Jeu ai vv. 426-457. Costui è da identificare molto probabilmente con Gregorio X, che aveva denunciato tale condotta al concilio di Lione del maggio-giugno 1274 e quindi l'aveva condannata con le decretali del novembre successivo, per poi morire il 10 gennaio $1276^{97}$. L'identificazione trova conferma nel fatto che nel Jeu risultano ancora vivi i ricchi banchieri Ermenfroi de Paris e Ermenfroi Crespin (vv. 218-219, 794-795), di cui il Nécrologe de la confrérie des jongleurs et des bourgeois d'Arras pone il decesso nel $1277^{98}$.

Inoltre, il testo appare contemporaneo all'esposizione del reliquiario di Notre-Dame cui si fa riferimento al v. 1077, occasione per

${ }^{96}$ Adam de la Halle, Teatro, Brusegan (ed.), ed. cit., p. 47.

${ }^{97}$ Jean Dufournet, Adam de la Halle..., ed. cit., p. 55.

${ }^{98}$ Roger Berger, Le Nécrologe de la confrérie des jongleurs et des bourgeois d'Arras (1194-1361), 2 vols. Arras, Impr. central de l'Artois 1963-1970, vol. II, 1970, p. 106. 
la venerazione dei fedeli che si ripeteva ogni anno per la Pentecoste e per i giorni seguenti.

Secondo Berger le Jeu de la Feuillée sarebbe stato scritto per una notte del Grand siège, ossia una solenne seduta della Confrérie ${ }^{99}$, che per l'anno 1276 dovette tenersi ad inizio giugno ${ }^{100}$, ed è allora questa data che va considerata il terminus post quem per la datazione di Aucassin et Nicolette.

D'altra parte anche l'altra opera teatrale di Adam de la Halle, le Jeu de Robin et Marion rappresentato alla corte angioina di Napoli tra il 1282 e il 1285, ma forse composto prima del Jeu de la Feuillée per il conte di Artois ${ }^{101}$, condivide elementi rilevanti con la chantefable a partire dalla centralità del genere pastorella e dello sperimentalismo formale praticato. Infatti in esso, con l'alternanza di dialoghi, versi e musica - come già rilevato -, l'autore drammatizza entrambi i tipi della pastorella, quella classica e quella oggettiva, peraltro sperimentando nuove soluzioni formali con la combinazione di due componenti che appaiono due strati testuali distinti, ossia il discorso lirico-citazione e il discorso dialogato infarcito di ulteriori citazioni liriche, per cui alla varietà metrica delle pastorelle citate, Adam oppone la regolarità del couplet ottosillabico e la rima mnemonica che salda la fine di una battuta con la battuta seguente ${ }^{102}$.

Colpisce infine la presenza anche nel Jeu de Robin et Marion dello sketch già ricordato nella chantefable, giocato sul significato metaforico da attribuire agli animali, questa volta attraverso un dialogo equivoco e denso di allusioni erotiche tra la pastora e il cavaliere a proposito di bestie incontrate da Marion e di cui va a caccia il cavaliere (vv. 30-50).

Il mondo di Arras e le opere del suo più illustre rappresentante Adam de la Halle appaiono allora punto di riferimento imprescindibile dell'anonimo di Aucassin et Nicolette, che si pone all'incrocio tra modalità narrative e liriche della tradizione cortese ormai prossima alla fine e modalità teatrali proprie al mondo borghese, che ne confermano la rappresentazione in scena.

La composizione della chantefable quindi si spiega appieno nella realtà arrageoise degli ultimissimi anni del xIII secolo (e di essa si sostanzia), quando era possibile e gradito al pubblico prendersi gioco della letteratura cortese che aveva concluso la sua parabola, perché ormai priva dei presupposti che ne avevano determinato la nascita felice e lo sviluppo rigoglioso.

\footnotetext{
${ }^{99}$ Roger Berger, Littérature et société, op. cit., p. 115.

${ }^{100}$ Pierre-Yves Badel (ed.), Adam de la Halle. Euvres complètes, Paris, Librairie Générale Française, 1995, p. 22

${ }^{101}$ Adam de la Halle, Teatro, Brusegan (ed.), ed. cit., p. 23.

${ }^{102}$ Ibid., pp. 25-26.
} 
Alla parodia però, come si è detto, corrisponde un messaggio positivo di tolleranza non solo letteraria, come implica la parodia stessa, ma ideologica.

Si è già osservato come la vicenda dei due protagonisti sia ambientata nel territorio occitanico, ove il riferimento a Biaucaire e al suo assedio rinvia senza dubbio alcuno alla crociata contro gli albigesi, come già rilevato da Griffin ${ }^{103}$.

Infatti la cittadina di Beaucaire, posta su un'alta rocca in posizione strategica sul Rodano, era stata infeudata a Simon de Montfort dall'arcivescovosco di Arles all'insaputa del papa. Quando comincia la ribellione del giovane conte Raimondo VII contro il proprio padre in partenza per la Spagna, essa prende il via appunto da Beaucaire, che resterà nella memoria collettiva un luogo densamente simbolico, non solo perché è proprio lì che avvenne il primo combattimento in larga scala tra i baroni meridionali e l'esercito di Simon de Montfort, ma soprattutto perché attaccata a sorpresa mentre costui è a Parigi, costituisce il primo rilevante successo di un assedio attuato dai ribelli ${ }^{104}$.

Solo a Beaucaire allora poteva compiersi la ribellione di Aucassin verso il proprio padre, in nome di un amore, quello cortese, espressione e motore della civiltà che si sarebbe spenta con la crociata contro gli Albigesi.

Non solo, ma l'anonimo sembra attuare un ulteriore rovesciamento proprio alla scrittura parodica: se, come suggerisce Uhl, il nome di Bougars che da venti anni assedia Beaucaire potrebbe significare 'bulgaro', 'sodomita' o 'eretico' 105 , esso tuttavia richiama in modo ironico quello di Bouchard de Marly, luogotenente di Simon de Montfort e sposo di sua cugina Alice de Montmorency, il quale si rivelerà suo fedele braccio destro in più occasioni, tanto da portare viveri e rinforzi a Simon assediato a Castelnauday nel 1211.

Se allora trova ulteriore conferma il sottinteso storico della chantefable Aucassin et Nicolette, tuttavia la messa in berlina di quella guerra che tanto ha nuociuto all'esperienza letteraria trobadorica va di pari passo con il riconoscimento e la rivendicazione del relativismo ideologico e culturale che vi è rappresentato.

Così Nicolette nonostante abbia scoperto la propria identità saracena, di cui assume anche l'aspetto esteriore tingendosi il volto con un'erba nel cap. XVIII ${ }^{106}$ - ciò che appare un'ulteriore divertente allusione alla tozeta mestissa marcabruniana -, abbandona Cartagena $\mathrm{e}$ risale la Spagna per ricongiungersi al cristiano Aucassin e affermare il diritto all'amore nonostante tutto; così soprattutto i generi letterari

\footnotetext{
${ }^{103}$ Robert Griffin, «Aucassin et Nicolette...», art. cit.

${ }^{104}$ Laurence W. Marvin, The Occitan War. A Military and Political History of the Albigensian Crusade, 1209-1218, Cambridge, University Press, 2008, pp. 234-257.

${ }^{105}$ Patrice Uhl, «Le Royaume de Torelore...», art. cit., p. 212.

${ }^{106}$ Così osserva Jacqueline de Weevers, «Nicolette's Blackness. Lost in Translation», Romance Notes, 34 (1993), pp. 317-325.
} 
e i motivi che li connotano e che si mantengono vitali nonostante e con il passare del tempo, vengono mirabilmente intrecciati grazie alla sapiente arte dell'anonimo per dialogare tra loro in nome della benefica e irriverente ironia, e finalmente diventare materia da reinterpretare alla luce delle nuove istanze sociali e culturali propri alla realtà borghese arrageoise di fine XIII secolo.

Ancora una volta quindi il riconoscimento del gioco intertestuale, particolarmente fecondo in un'opera che di quello si è nutrita come Aucassin et Nicolette, permette non solo di individuare connessioni sinora sfuggite con testi remoti come quella con la pastorella maggiore di Marcabruno e la lirica del gatto rosso di Guglielmo IX, ma estendendosi anche a un'opera più recente come Le Jeu de la Feuillée, ha permesso di avanzare una nuova ipotesi di datazione della chantefable alla fine del Duecento, ciò che consente allora di illuminare pienamente il ricorso alla parodia da parte dell'anonimo perché attuato alla fine dell'esperienza cortese, che viene appunto riassunta e diversamente declinata nella sua complessa varietà di generi, stili, motivi. 


\title{
$\cos$
}

\section{DE GRANT MAL AMALADIS E LA PASTORELLA NASCOSTA \\ IN AUCASSIN ET NICOLETTE: \\ UNA PROPOSTA DI DATAZIONE DELLA CHANTEFABLE}

\begin{abstract}
Riassunto: L'anonima chantefable Aucassin et Nicolette, fondata sulla parodia, ha una controversa datazione. Tuttavia la presenza di l'autrier - marca lessicale identificativa del genere pastorella -, e una serie di allusioni erotiche autorizzano a rileggere con maggiore attenzione il cap. XI, e in particolare il v. 21 che riproduce il v. 12 del prologo, a conferma della centralità della scena rappresentata, in connessione sia con la lirica del gatto rosso di Guglielmo IX, sia con la pastorella maggiore di Marcabruno ad essa connessa. Non solo, ma l'analisi lessicale di un altro vocabolo dello stesso capitolo consente di cogliere un ulteriore gioco intertestuale con le Jeu de la Feuillée di Adam de la Halle, che fornisce il 1276 come terminus post quem per la datazione della chantefable e di porre la sua composizione nell'ambiente borghese di Arras, di cui condivide le istanze sociali e culturali declinate attraverso il ricorso alla parodia.
\end{abstract}

PAROle Chiave: datazione di Aucassin et Nicolette, parodia, intertestualità, genere pastorella, Jeu de la Feuillée di Adam de la Halle.

\section{DE GRANT MAL AMALADIS AND THE HIDDEN PASTORELLA IN AUCASSIN AND NICOLETTE: A PROPOSAL FOR DATING THE CHANTEFABLE}

\begin{abstract}
The anonymous chantefable Aucassin et Nicolette, based on parody, has a controversial dating. However the presence of the l'autrier - that identifyes the genre of pastorella - and a series of erotic allusions authorize us to read again the chap. XI, and in particular v. 21 which reproduces the v. 12 of the prologue, confirming the centrality of the scene represented, in connection both with the lyric of the red cat of William IX, and with the Marcabruno's most important pastorella, connected to it. Not only that, but the lexical analysis of another word in the same chapter allows us to grasp a further intertextual game with Adam de la Halle's Jeu de la Feuillée, which provides 1276 as a terminus post quem for the chantefable dating and to place its composition in the bourgeois environment of Arras, of which it shares the social and cultural needs declined through the use of parody.
\end{abstract}

KEYWORDS: dating of Aucassin et Nicolette, parody, intertextuality, genre pastorella, Adam de la Halle's Jeu de la Feuillée. 